\title{
Multicomponent mixture of charged hard-sphere chain molecules in the polymer mean-spherical approximation
}

\author{
Yu. V. Kalyuzhnyi \\ Institute for Condensed Matter Physics, Svientsitskoho 1, 290011 Lviv, Ukraine \\ P. T. Cummings \\ Department of Chemical Engineering, University of Tennessee, Knoxville 37996-2200, USA
}

Running title: Charged hard-sphere chain fluid

The address for correspondence: Institute for Condensed Matter Physics, Svientsitski Str. 1, 79011 Lviv, UKRAINE; e-mail: yukal@icmp.lviv.ua 


\begin{abstract}
The analytical solution of the recently proposed ideal chain polymer mean-spherical approximation (Yu.Kalyuzhnyi, Mol.Phys., 94, $735(1998)$ ) is presented for the multicomponent mixture of charged hardsphere linear chain flexible molecules. The solution apply to any mixture of chain molecules with arbitrary distribution of the charge and size of the beads along the molecular backbone. Closed form analytical expressions for the internal energy, Helmholtz free energy, chemical potentials and pressure are derived. By way of illustration thermodynamical properties of several versions of the fluid of charged chain molecules of different length, including the molecules with uniform, diblock and alternating distribution of the charge are studied. Theoretical predictions are in reasonable agreement with available computer simulation predictions. We present also the liquid-gas phase diagrams for the systems with diblock and alternating distribution of the charge.
\end{abstract}




\section{INTRODUCTION}

In recent years substantial amout of efforts has been focused on the development of theoretical approaches predicting the equilibrium structure and thermodynamical properties of charged polymer solutions. This information enables one to esteblish the stability limits and to build the phase diagrams of such fundamentally and technologically importent systems as polyelectrolyte solutions (for example DNA, polyacrilic acid) and polyampholyte solutions (for example proteins). One of the simplest continuum models of charged polymer solutions is represented by the fluid of charged hard-sphere linear chain flexible molecules. In spite of its simplicity this model incorporates several essential features of charged polymer fluids, such as excluded volume effects, chain connectivity and flexibility, distribution of the charge along the chain backbone and Coulomb interaction. Recently several off-lattice theories for the fluid of flexible charged chain molecules, based on the integral-equation techniques developed for the fluids of small molecules, have been proposed. These include extension [1] of the polymer reference interaction site model (RISM) integral equation theory of Schweizer and Curro [2], extension [3, 4 ] of the simple interpolation scheme (SIS) of Stell and Zhou [5] and extensions [6 10] of the multidensity integral equation theory for associating fluids of Wertheim [11]. The latter studies are based on the analytical solution of the mean spherical approximation (MSA) version of the multidensity theory, the so-called polymer MSA (PMSA) [6 8, 12]. In the case of uncharged system direct application of Wertheim's original formulation by Chang and Sandler [13, 14] gave rise to a polydisperse fluid of chains with a prescribe mean number of beads. A subsequent extension by Kalyuzhnyi and Cummings [15] yields a model that polymerizes to a fluid of chains of fixed length in the complete association limit. In that limit, two-density Wertheim's Ornstein-Zernike (OZ) equation becomes identical to the Rossky-Chiles [16] version of the "proper" site-site integral equations [17] ("proper-RISM") as first noted by Kalyuzhnyi and Stell [18] and later discussed in more details by Stell [19] and by Kalyuzhnyi and Cummings [20]. More recently complete associ-

ation version of this extension was combined with the ideal chain approximation [11, 13, 21] 
and generalized for the systems with arbitrary long-range potential outside the hard-core region [9]. As a result the corresponding version of the PMSA was formulated and Høye-Stell scheme [22] of calculating the simple fluids thermodynamical properties was extended in the frames of the PMSA [9]. Subsequently von Solms and Chiew [10] combine PMSA and ideal chain approximation in a way similar to that proposed by Chang and Sandler [13]. The resulting polydisperse chain mixture was used to describe thermodynamical properties of the two-component mixture of fixed-length chain molecules with equally charged beads and oppositelly charged monomer counterions. Results of the theory appeare to be in reasonable agreement with computer simulation results [23]. We note in passing, that ideal chain approximation utilized by von Solms and Chiew [10] is somewhat different from the original one [11,13]. In the corresponding Wertheim's OZ equation the authors take into account all partial correlation functions, while due the original ideal chain approximation [11,13] correlations involving at least one doubly bonded particle are neglected. More recently general solution of the PMSA [7,8] for polymerizing charged hard spheres, supplemented by the ideal chain approximation, was used to derive closed form analytical expressions for thermodynamical properties of charged chain fluid with a certain restrictions imposed on the sizes of the chain beads [24]. In the latter study the authors propose an improved version of the ideal chain approximation, which satisfies the Debye-Hückel limiting law.

In this paper we present an analytical solution of the ideal chain PMSA [9] for the multicomponent mixture of charged hard-sphere flexible chain molecules. Unlike the previous studies proposed version of the PMSA is quite general and apply for any mixture of the chain molecules with arbitrary distribution of the charge and size of the beads along the molecular backbone. Our solution represents a complete association limit of the general solution obtained earlier [7,8] for the fluid of polymerizing charged hard spheres. Similar, as in the case of primitive electrolyte model [25] this solution reduces to the solution of only one algebraic equation for the MSA-like screening parameter $\Gamma$. We also generalize recently developed method of calculating PMSA thermodynamics via the energy route [9] for the multicomponent case and derived closed form analytical expressions for internal energy, 
Helmholtz free energy, chemical potentials and pressure in terms of the present solution. By way of illustration we consider several different versions of the fluid of charged hard-sphere chain molecules, including the molecules with uniform, diblock and alternating distribution of the charge along the chain backbone.

The paper is orgenized as follows. In the next section we discuss detailes of the model to be studied and formulate ideal chain PMSA theory. Section III gives solution of the ideal chain PMSA and Section IV contains expressions for thermodynamic and structure properties of the system. Numerical results and discussion can be found in Section $\mathrm{V}$ and in Section VI we collect our conclusions.

\section{THE MODEL AND IDEAL CHAIN PMSA THEORY}

The model fluid we consider consists of the $M$-component mixture of freely jointed tangent hard-sphere chain molecules with each molecule of species $a$ represented by $m_{a}$ charged hard-sphere sites of sizes $\sigma_{\alpha}^{a}$ and charges $e z_{\alpha}^{a}$. The total number density of the system is $\rho=\sum_{a} \rho_{a}$, where $\rho_{a}$ is the number density of species $a$, and we assume charged neutrality conditions

$$
\sum_{a \alpha} \rho_{a} z_{\alpha}^{a}=0
$$

We denote the molecular species by the small letters $a, b, c, \ldots$ taking the values $1,2, \ldots, M$ and the site type in a given molecule $a$ by a small greek letters $\alpha, \beta, \gamma, \ldots$, which take the values $1,2, \ldots, m_{a}$. Thus the site has two indices, one denoting the species of the molecule and the other the site type in the molecule. For example the hard-sphere diameter of the site of type $\alpha$ belonging to the molecule of the species $a$ is denoted by $\sigma_{\alpha}^{a}$.

The site-site pair potential $U_{\alpha \beta}^{a b}(r)$ between the sites of the type $\alpha$ and $\beta$ belonging to the molecules of species $a$ and $b$ can be written in the form

$$
U_{\alpha \beta}^{a b}(r)=U_{\alpha \beta}^{(h s) a b}(r)+U_{\alpha \beta}^{(C) a b}(r)
$$

where $U_{\alpha \beta}^{(h s) a b}(r)$ is the hard-sphere potential and $U_{\alpha \beta}^{(C) a b}(r)$ is the Coulomb potential 


$$
U_{\alpha \beta}^{(C) a b}(r)=\frac{e^{2} z_{\alpha}^{a} z_{\beta}^{b}}{\epsilon_{0} r}
$$

with $\epsilon_{0}$ being the dielectric constant of the continuum.

In this study we are using the multidensity version of the mean spherical approximation (MSA) [11,12,6,9] (or polymer MSA (PMSA)) supplemented by the so-called ideal chain approximation [11, 13,21,9]. Both PMSA and the ideal chain approximation have been discussed at length earlier and therefore we will omit any details and present here only the final expressions. The theory consists of the Ornstein-Zernike-like integral equation

$$
\hat{\mathbf{h}}_{\alpha \beta}^{a b}(k)=\hat{\mathbf{c}}_{\alpha \beta}^{a b}(k)+\sum_{c} \rho_{c} \sum_{\gamma} \hat{\mathbf{c}}_{\alpha \gamma}^{a c}(k) \boldsymbol{\alpha} \hat{\mathbf{h}}_{\gamma \beta}^{c b}(k)
$$

and PMSA boundary conditions

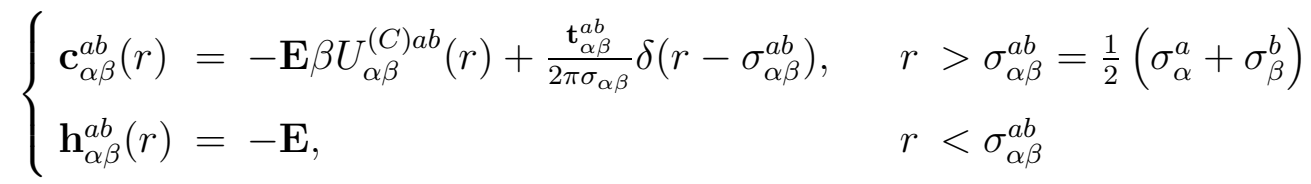

where $\hat{\mathbf{h}}_{\alpha \beta}^{a b}(k)$ and $\hat{\mathbf{c}}_{\alpha \beta}^{a b}(k)$ are the matrices with the elements being the Fourier transforms of the elements of the matrices $\mathbf{h}_{\alpha \beta}^{a b}(r)$ and $\mathbf{c}_{\alpha \beta}^{a b}(r)$

$$
\mathbf{h}_{\alpha \beta}^{a b}(r), \mathbf{c}_{\alpha \beta}^{a b}(r)=\left(\begin{array}{ccc}
c_{\alpha_{0} \beta_{0}}^{a b}(r) & c_{\alpha_{0} \beta_{A}}^{a b}(r) & c_{\alpha_{0} \beta_{B}}^{a b}(r) \\
c_{\alpha_{A} \beta_{0}}^{a b}(r) & c_{\alpha_{A} \beta_{A}}^{a b}(r) & c_{\alpha_{A} \beta_{B}}^{a b}(r) \\
c_{\alpha_{B} \beta_{0}}^{a b}(r) & c_{\alpha_{B} \beta_{A}}^{a b}(r) & c_{\alpha_{B} \beta_{B}}^{a b}(r)
\end{array}\right),
$$

$\mathbf{t}_{i j}, \boldsymbol{\alpha}$ and $\mathbf{E}$ are the following matrices

$$
t_{\alpha_{i} \beta_{j}}^{a b}=\frac{\delta_{a b}}{2 \rho_{a}}\left[\delta_{i A} \delta_{j B} \frac{\delta_{\alpha \beta+1}}{\sigma_{\alpha \alpha-1}}+\delta_{i B} \delta_{j A} \frac{\delta_{\alpha \beta-1}}{\sigma_{\alpha \alpha+1}}\right], \quad \alpha_{i j}=1-\delta_{i j}+\delta_{0 i} \delta_{0 j}, \quad E_{i j}=\delta_{0 i} \delta_{0 j} .
$$

Here the lower indices $i, j$ each take the values $0, A$, and $B$, and denote the bonding states of the corresponding particle, i.e. 0 denotes the unbonded state and $A$ and $B$ denote the $A$-bonded and $B$-bonded states, respectively. The total partial correlation functions $h_{\alpha_{i} \beta_{j}}^{a b}(r)$ are related to the site-site total correlation functions $h_{\alpha \beta}^{a b}(r)$ by the following relation

$$
h_{\alpha \beta}^{a b}(r)=\sum_{i j} h_{\alpha_{i} \beta_{j}}(r)
$$

The set of the OZ equations (4) together with the PMSA closure conditions (5) represent our ideal chain PMSA theory for the fluid of charged linear chain hard-sphere molecules. 


\section{SOLUTION OF THE IDEAL CHAIN PMSA FOR THE FLUID OF CHARGED CHAIN MOLECULES}

Recently a general solution of the PMSA for the multicomponent mixture of polymerizing charged hard spheres was published [7,8]. Here we elaborate on this general solution by utilizing additionally the ideal chain approximation [11,13,9] and specializing it for the case of the model at hand.

The general solution was obtained using Baxter's tecnique, which factorizes the initial OZ equation (4) into a set of two equations

$$
\begin{gathered}
\mathbf{S}_{\alpha \beta}^{a b}(|r|)=\mathbf{Q}_{\alpha \beta}^{a b}(r)-\sum_{c} \rho_{c} \sum_{\gamma} \int d r^{\prime} \mathbf{Q}_{\alpha \gamma}^{a c}\left(r^{\prime}\right) \boldsymbol{\alpha}\left[\mathbf{Q}_{\beta \gamma}^{b c}\left(r^{\prime}-r\right)\right]^{T} \\
\mathbf{J}_{\alpha \beta}^{a b}(|r|)=\mathbf{Q}_{\alpha \beta}^{a b}(r)+\sum_{c} \rho_{c} \sum_{\gamma} \int d r^{\prime} \mathbf{J}_{\alpha \gamma}^{a c}\left(\left|r^{\prime}-r\right|\right) \boldsymbol{\alpha} \mathbf{Q}_{\gamma \beta}^{c b}\left(r^{\prime}\right)
\end{gathered}
$$

where $T$ denotes the transpose matrix. The projections $\mathbf{S}_{\alpha \beta}^{a b}(r)$ and $\mathbf{J}_{\alpha \beta}^{a b}(r)$

$$
\mathbf{S}_{\alpha \beta}^{a b}(r)=2 \pi \int_{r}^{\infty} d r^{\prime} r^{\prime} \mathbf{c}_{\alpha \beta}^{a b}\left(r^{\prime}\right), \quad \mathbf{J}_{\alpha \beta}^{a b}(r)=2 \pi \int_{r}^{\infty} d r^{\prime} r^{\prime} \mathbf{h}_{\alpha \beta}^{a b}\left(r^{\prime}\right)
$$

satisfy the following boundary conditions

$$
\begin{cases}\mathbf{J}_{\alpha \beta}^{a b}(r)=\pi r^{2} \mathbf{E}+\mathbf{J}_{\alpha \beta}^{a b}, & r \leq \sigma_{\alpha \beta}^{a b} \\ \mathbf{S}_{\alpha \beta}^{a b}(r)=-\frac{\beta e^{2}}{\epsilon_{0}} \frac{z_{\alpha}^{a} z_{\beta}^{b} e^{-\mu|r|}}{\mu} \mathbf{E}, & r>\sigma_{\alpha \beta}^{a b}\end{cases}
$$

obtained from (5). Here $\mathbf{J}_{\alpha \beta}^{a b}=\mathbf{J}_{\alpha \beta}^{a b}(0)$ and the limit $\mu \rightarrow 0$ is to be taken at the end of the calculations.

From the analysis of the equations (7) (8) we get the following expression for the factor function $\mathbf{Q}_{\alpha \beta}^{a b}(r)$

$$
\mathbf{Q}_{\alpha \beta}^{a b}(r)=\theta\left(\sigma_{\alpha \beta}^{a b}-r\right)\left[\mathbf{q}_{\alpha \beta}^{a b}(r)+\mathbf{t}_{\alpha \beta}^{a b}\right]-\left(\tilde{\mathbf{z}}_{\alpha}^{a}\right)^{T} \tilde{\mathbf{a}}_{\beta}^{b}, \quad r>\lambda_{\alpha \beta}^{a b}
$$

where $\lambda_{\alpha \beta}^{a b}=\left(\sigma_{\alpha}^{a}-\sigma_{\beta}^{b}\right) / 2$ and the function $\mathbf{q}_{\alpha \beta}^{a b}(r)$ is defined in the range $\lambda_{\alpha \beta}^{a b}<r<\sigma_{\alpha \beta}^{a b}$ by

$$
\mathbf{q}_{\alpha \beta}^{a b}(r)=\frac{1}{2} \tilde{\mathbf{E}}^{T} \tilde{\mathbf{A}}_{\beta}^{b}\left(r-\sigma_{\alpha \beta}^{a b}\right)\left(r-\lambda_{\beta \alpha}^{b a}\right)+\beta_{\alpha \beta}^{a b}\left(r-\sigma_{\alpha \beta}^{a b}\right) .
$$


Here $\tilde{\mathbf{E}}, \tilde{\mathbf{a}}_{\alpha}^{a}$ and $\tilde{\mathbf{z}}_{\alpha}^{a}$ are the row vectors

$$
\tilde{\mathbf{E}}=(1,0,0), \quad \tilde{\mathbf{a}}_{\alpha}^{a}=\left(a_{\alpha_{0}}^{a}, a_{\alpha_{A}}^{a}, a_{\alpha_{B}}^{a}\right), \quad \tilde{\mathbf{z}}_{\alpha}^{a}=\left(z_{\alpha}^{a}, 0,0\right)
$$

Coefficients of the Baxter $q$-function can be expressed in terms of only one parameter, i.e. the screening PMSA parameter $\Gamma$ [25, 7, 8]. We have

$$
\begin{aligned}
& \beta_{\alpha_{i} \beta_{j}}^{a b}=\delta_{i 0} \delta_{j 0} \frac{\pi \sigma_{\beta}^{b}}{\Delta}+\frac{2 \pi \beta^{*}}{\sigma_{\alpha}^{a} \Gamma}\left(X_{\alpha_{0}}^{a}-\delta_{i 0} z_{\alpha}^{a}\right) X_{\beta_{j}}^{b}, \\
& A_{\beta_{j}}^{b}=\frac{4 \pi \beta^{*}}{\Gamma} \eta^{B} X_{\beta_{j}}^{b}+\delta_{j 0} \frac{2 \pi}{\Delta}\left[1+\zeta_{2} \sigma_{\beta}^{b} \frac{\pi}{2 \Delta}\right]-\frac{\pi}{\Delta}\left[\left(1-\delta_{\beta m_{a}}\right) \frac{\delta_{j B} \sigma_{\beta+1}^{b}}{\sigma_{\beta+1 \beta}^{b b}}+\left(1-\delta_{\beta 1}\right) \frac{\delta_{j A} \sigma_{\beta-1}^{b}}{\sigma_{\beta-1 \beta}^{b b}}\right] \\
& \Delta=1-\frac{1}{6} \pi \zeta_{3}, \quad \zeta_{n}=\sum_{d} \rho_{d} \sum_{k}\left(\sigma_{k}^{d}\right)^{n}, \quad \chi_{2}=\sum_{d} \rho_{d} \sum_{k} z_{k}^{d}\left(\sigma_{k}^{d}\right)^{2} \\
& a_{\alpha_{i}}^{a}=2 \pi \beta^{*} X_{\alpha_{i}}^{a} / \Gamma \\
& X_{\alpha_{0}}^{a}=\left[z_{\alpha}^{a}-\eta^{B}\left(\sigma_{\alpha}^{a}\right)^{2}\right] \Gamma_{\alpha}^{a}, \quad X_{\alpha_{i}}^{a}=\sigma_{\alpha}^{a}\left[\tau_{\alpha_{i}}^{a}(z)-\eta^{B} \tau_{\alpha_{i}}^{a}\left(\sigma^{2}\right)\right], \quad(i \neq 0) \\
& \eta^{B}=\frac{\frac{\pi}{2 \Delta} \sum_{d} \rho_{d} \sum_{\gamma} \sigma_{\gamma}^{d}\left\{z_{\gamma}^{d} \Gamma_{\gamma}^{d}+\sigma_{\gamma}^{d}\left[\tau_{\gamma_{A}}^{d}(z)+\tau_{\gamma_{B}}^{d}(z)\right]\right\}}{1+\frac{\pi}{2 \Delta} \sum_{d} \rho_{d} \sum_{\gamma}\left(\sigma_{\gamma}^{d}\right)^{2}\left[\sigma_{\gamma}^{d} \Gamma_{\gamma}^{d}+\tau_{\gamma_{A}}^{d}\left(\sigma^{2}\right)+\tau_{\gamma_{B}}^{d}\left(\sigma^{2}\right)\right]}, \quad \Gamma_{\alpha}^{a}=\left(1+\sigma_{\alpha}^{a} \Gamma\right)^{-1} \\
& \tau_{\alpha_{A}}^{a}(y)=\frac{1}{2} \sum_{\gamma=2}^{\alpha} \Gamma_{\gamma-1}^{a} \rho_{a}^{\alpha-\gamma} \frac{y_{\gamma-1}^{a}}{\sigma_{\gamma \gamma-1}^{a a}} \prod_{\delta=\gamma}^{\alpha} \Gamma_{\delta}^{a}\left[2^{\gamma-\alpha}\left(1-\delta_{\gamma \alpha}\right) \prod_{\omega=\gamma+1}^{\alpha} \frac{\sigma_{\omega-1}^{a}}{\sigma_{\omega \omega-1}^{a}}+\delta_{\gamma \alpha}\right]\left(1-\delta_{\alpha 1}\right) \\
& \tau_{\alpha_{B}}^{a}(y)=\frac{1}{2} \sum_{\gamma=\alpha}^{m_{a}-1} \Gamma_{\gamma+1}^{a} \rho_{a}^{\gamma-\alpha} \frac{y_{\gamma+1}^{a}}{\sigma_{\gamma \gamma+1}^{a a}} \prod_{\delta=\alpha}^{\gamma} \Gamma_{\delta}^{a}\left[2^{\alpha-\gamma}\left(1-\delta_{\gamma \alpha}\right) \prod_{\omega=\alpha}^{\gamma-1} \frac{\sigma_{\omega+1}^{a}}{\sigma_{\omega \omega+1}^{a}}+\delta_{\gamma \alpha}\right]\left(1-\delta_{\alpha m_{a}}\right)
\end{aligned}
$$

Here $\Gamma$ satisfies the following equation

$$
\Gamma^{2}=\pi \beta^{*} \sum_{a \alpha} \rho_{a} \sum_{\alpha} \tilde{\mathbf{X}}_{\alpha}^{a} \boldsymbol{\alpha}\left(\tilde{\mathbf{X}}_{\alpha}^{a}\right)^{T}
$$

where $\tilde{\mathbf{X}}_{\alpha}^{a}$ is the row vector $\tilde{\mathbf{X}}_{\alpha}^{a}=\left(X_{\alpha_{0}}^{a}, X_{\alpha_{A}}, X_{\alpha_{B}}\right)$.

Thus solution of the PMSA for the present model reduces to the solution of the algebraic equation (21) for the screening parameter $\Gamma$. 


\section{STRUCTURE AND THERMODYNAMICS}

\section{A. Structure properties}

The contact values of the regular part of the partial pair distribution functions $g_{\alpha_{i} \beta_{j}}^{a b}\left(\sigma_{\alpha \beta}^{a b}+\right)$ follow from the relation (8) after differentiating it with respect to $r$ and taking the limit of $r \rightarrow \sigma_{\alpha \beta}^{a b}+$

$$
2 \pi \sigma_{\alpha \beta}^{a b} g_{\alpha_{i} \beta_{j}}^{a b}\left(\sigma_{\alpha \beta}^{a b}+\right)=2 \pi \delta_{0 i} \delta_{j 0} \sigma_{\alpha \beta}^{a b} g_{\alpha \beta}^{(h s) a b)}\left(\sigma_{\alpha \beta}^{a b}+\right)-2 \pi \beta^{*} X_{\alpha_{i}}^{a} X_{\beta_{j}}^{b}+T_{\alpha_{i} \beta_{j}}^{a b}
$$

where $g_{\alpha \beta}^{(h s) a b}\left(\sigma_{\alpha \beta}^{a b}+\right)$ is the hard-sphere contact values and

$$
\begin{gathered}
T_{\alpha_{i} \beta_{j}}^{a b}=\delta_{a b}\left\{\left[\frac{\left(1-\delta_{\alpha m_{a}}\right)\left(1-\delta_{\alpha m_{a}-1}\right) \delta_{i B} \delta_{j A} \delta_{\alpha \beta-2}}{4 \rho_{a} \sigma_{\alpha \alpha+1}^{a a} \sigma_{\alpha+1 \beta}^{a a}}+\frac{\left(1-\delta_{\alpha 1}\right)\left(1-\delta_{\alpha 2}\right) \delta_{i A} \delta_{j B} \delta_{\alpha \beta+2}}{4 \rho_{a} \sigma_{\alpha \alpha-1}^{a a} \sigma_{\alpha-1 \beta}^{a a}}\right]+\right. \\
\frac{\pi}{2 \Delta} \delta_{j 0} \sigma_{\beta}^{a}\left[\left(1-\delta_{\alpha m_{a}}\right) \delta_{i B} \frac{\sigma_{\alpha+1}^{a}}{\sigma_{\alpha \alpha+1}^{a a}}+\left(1-\delta_{\alpha 1}\right) \delta_{i A} \frac{\sigma_{\alpha-1}^{a}}{\sigma_{\alpha \alpha-1}^{a a}}\right]+ \\
\left.\frac{\pi}{2 \Delta} \delta_{i 0} \sigma_{\alpha}^{a}\left[\left(1-\delta_{\beta m_{a}}\right) \delta_{j B} \frac{\sigma_{\beta+1}^{a}}{\sigma_{\beta+1 \beta}^{a a}}+\left(1-\delta_{\beta 1}\right) \delta_{j A} \frac{\sigma_{\beta-1}^{a}}{\sigma_{\beta-1 \beta}^{a a}}\right]\right\}
\end{gathered}
$$

The values of $g_{\alpha_{i} \beta_{j}}^{a b}(r)$ for $r>\sigma_{\alpha \beta}^{a b}$ can be calculated using the following relation

$$
g_{\alpha_{i} \beta_{j}}^{a b}(r)=\delta_{0 i} \delta_{0 j}+\frac{1}{2 \pi^{2} r} \int_{0}^{\infty}\left[\hat{\gamma}_{\alpha_{i} \beta_{j}}^{a b}(k)-\delta_{i 0} \delta_{j 0} \beta \hat{U}_{\alpha \beta}^{(C) a b}(k)\right] k \sin (k r) d k
$$

where $\hat{U}_{\alpha \beta}^{(C) a b}(k)$ is the Fourier transform of the Coulomb potential $U_{\alpha \beta}^{(C) a b}(r)$ and expression for the function $\hat{\gamma}_{\alpha_{i} \beta_{j}}^{a b}(k)=\hat{h}_{\alpha_{i} \beta_{j}}^{a b}(k)-\hat{c}_{\alpha_{i} \beta_{j}}^{a b}(k)$ follows from the set of equations (77) and (8), written in the Fourier $k$-space

$$
\left\{\begin{array}{l}
\boldsymbol{\rho}^{-1}-\hat{\mathbf{c}}(k)=\hat{\mathbf{Q}}(k) \boldsymbol{\rho} \hat{\mathbf{Q}}^{T}(-k) \\
\boldsymbol{\rho}^{-1}+\hat{\mathbf{h}}(k)=\left[\boldsymbol{\rho} \hat{\mathbf{Q}}(k) \boldsymbol{\rho} \hat{\mathbf{Q}}^{T}(-k) \boldsymbol{\rho}\right]^{-1}
\end{array},\right.
$$

which gives

$$
\hat{\gamma}(k)=\hat{\mathbf{Q}}(k) \boldsymbol{\rho} \hat{\mathbf{Q}}^{T}(-k)+\left[\boldsymbol{\rho} \hat{\mathbf{Q}}(k) \boldsymbol{\rho} \hat{\mathbf{Q}}^{T}(-k) \boldsymbol{\rho}\right]^{-1}-2 \boldsymbol{\rho}^{-1}
$$

Here $\boldsymbol{\rho}, \hat{\mathbf{h}}(k), \hat{\mathbf{c}}(k)$ and $b Q(k)$ are the matrices with the elements $\rho_{\alpha_{i} \beta_{j}}^{a b}=\delta_{a b} \delta_{\alpha \beta} \alpha_{i j} \rho_{a}, \hat{h}_{\alpha_{i} \beta_{j}}^{a b}(k)$, $\hat{c}_{\alpha_{i} \beta_{j}}^{a b}(k)$ and 


$$
\hat{Q}_{\alpha_{i} \beta_{j}}^{a b}(k)=\left[\boldsymbol{\rho}^{-1}\right]_{\alpha_{i} \beta_{j}}^{a b}-\int_{\lambda_{\beta \alpha}}^{\infty} d r e^{i k r} Q_{\alpha_{i} \beta_{j}}^{a b}(r)
$$

respectively. Substituting expression for the fuction $Q_{\alpha_{i} \beta_{j}}^{a b}(r)$ (11) into the right hand side of the Fourier transformation (27) we find that

$$
\begin{gathered}
\hat{\mathbf{Q}}_{\alpha \beta}^{a b}(k)=\delta_{a b} \delta_{\alpha \beta}\left(\rho_{a} \boldsymbol{\alpha}\right)^{-1}-\left\{\tilde{\mathbf{E}} \tilde{\mathbf{A}}_{\beta}^{b} \varphi_{2}\left(k, \sigma_{\alpha}^{a}\right)+\left(\boldsymbol{\beta}_{\alpha \beta}^{a b}-\frac{1}{2} \tilde{\mathbf{E}}_{\beta}^{b} \sigma_{\alpha}^{a}\right) \varphi_{1}\left(k, \sigma_{\alpha}^{a}\right)+\right. \\
\left.+\left(\boldsymbol{\beta}_{\alpha \beta}^{a b} \sigma_{\alpha}^{a}-\mathbf{t}_{\alpha \beta}^{a b}\right) \varphi_{0}\left(k, \sigma_{\alpha}^{a}\right)-\left(\tilde{\mathbf{z}}_{\alpha}^{a}\right)^{T} \tilde{\mathbf{a}}_{\beta}^{b} \frac{i}{k}\right\}
\end{gathered}
$$

where

$$
\begin{gathered}
\varphi_{2}(k, \sigma)=-\frac{1}{k^{3}}\left[\left(\frac{1}{2} k^{2} \sigma^{2}+i k \sigma-1\right) e^{i k \sigma}+1\right], \quad \varphi_{1}=\frac{1}{k^{2}}\left[(1-i k \sigma) e^{i k \sigma}-1\right], \\
\varphi_{0}(k, \sigma)=\frac{i}{k}\left(e^{i k \sigma}-1\right)
\end{gathered}
$$

This result allow us to derive the expression for the structure factor

$$
\hat{S}_{\alpha \beta}^{a b}(k)=\delta_{a b} \delta_{\alpha \beta}+\sqrt{\rho_{a} \rho_{b}} \hat{h}_{\alpha \beta}^{a b}(k)
$$

Using the second of the relations (25) we have

$$
\hat{S}_{\alpha \beta}^{a b}(k)=\frac{1}{\sqrt{\rho_{a} \rho_{b}}}\left\{\left[\hat{\mathbf{Q}}(k) \boldsymbol{\rho} \hat{\mathbf{Q}}^{T}(-k)\right]^{-1}\right\}_{\alpha_{0} \beta_{0}}^{a b}
$$

\section{B. Thermodynamic properties}

Expression for the excess internal energy $\Delta E$ follows from the standard relation

$$
\frac{\Delta E}{V}=2 \pi \sum_{a b} \rho_{a} \rho_{b} \sum_{\alpha \beta} \int_{0}^{\infty} d r r^{2} U_{\alpha \beta}^{(C) a b}(r) g_{\alpha \beta}^{a b}(r),
$$

which after some calculations gives

$$
\beta \frac{\Delta E}{V}=\beta^{*} \sum_{a} \rho_{a} \sum_{\alpha} z_{\alpha}^{a} N_{\alpha}^{a}
$$

where $\sigma_{\alpha}^{a} N_{\alpha}^{a}=\sum_{i=0}^{B} X_{\alpha_{i}}^{a}-z_{\alpha}^{a}$ 
The other thermodynamical properties of the system in question can be calculated following the method developed earlier [9]. This method generalizes Høye-Stell MSA energy route to thermodynamics [22] in the frames of the present PMSA approach. Originally this generalization was developed for the one-component case. Extension of the method in the multicomponent case is rather straightforward and yields the following expressions for the Helmholtz free energy $A$, pressure $P$ and chemical potential $\mu_{\alpha}^{a}$ in excess to their reference system values

$$
\begin{gathered}
-\beta \frac{A-A^{(r e f)}}{V}=J+\frac{1}{2} \sum_{a b} \rho_{a} \rho_{b} \sum_{\alpha \beta}\left\{\boldsymbol{\alpha}\left[\hat{\mathbf{c}}_{\alpha \beta}^{a b}-\hat{\mathbf{c}}_{\alpha \beta}^{(0) a b}\right] \boldsymbol{\alpha}\right\}_{00}-\beta \frac{\Delta E}{V}+ \\
+\frac{1}{3} \pi \sum_{a b} \rho_{a} \rho_{b} \sum_{\alpha \beta}\left(\sigma_{\alpha \beta}^{a b}\right)^{3} \operatorname{Tr}\left[\mathbf{g}_{\alpha \beta}^{a b} \boldsymbol{\alpha} \mathbf{g}_{\beta \alpha}^{b a} \boldsymbol{\alpha}-\mathbf{g}_{\alpha \beta}^{(0) a b} \boldsymbol{\alpha} \mathbf{g}_{\beta \alpha}^{(0) b a} \boldsymbol{\alpha}\right]- \\
-\frac{1}{3} \sum_{a b} \rho_{a} \rho_{b} \sum_{\alpha \beta}\left(\sigma_{\alpha \beta}^{a b}\right)^{2} \operatorname{Tr}\left\{\mathbf{t}_{\alpha \beta}^{a b} \boldsymbol{\alpha}\left[\partial \mathbf{g}_{\alpha \beta}^{a b}-\partial \mathbf{g}_{\alpha \beta}^{(0) a b}\right] \boldsymbol{\alpha}\right\}, \\
\beta\left(P-P^{(r e f)}\right)=-\beta \frac{A-A^{(r e f)}}{V}-\frac{1}{2} \sum_{a b} \rho_{a} \rho_{b} \sum_{\alpha \beta}\left\{\boldsymbol{\alpha}\left[\hat{\mathbf{c}}-\hat{\mathbf{c}}^{(0)}\right] \boldsymbol{\alpha}\right\}_{00}+\beta \frac{\Delta}{V} \\
-\beta \rho_{a}\left(\mu_{\alpha}^{a}-\mu_{\alpha}^{(r e f) a}\right)=\frac{1}{2} \rho_{a} \sum_{b} \rho_{b} \sum_{\beta}\left\{\boldsymbol{\alpha}\left[\hat{\mathbf{c}}_{\alpha \beta}^{a b}-\hat{\mathbf{c}}_{\alpha \beta}^{(0) a b}\right] \boldsymbol{\alpha}\right\}_{00}-\beta \frac{\Delta E_{\alpha}^{a}}{V}
\end{gathered}
$$

where the quantities with the superscript (ref) denote the reference system quantities and the quantities with superscript (0) denote the corresponding zero charge PMSA quantities

$$
\begin{gathered}
\hat{\mathbf{c}}=\hat{\mathbf{c}}(k=0), \quad \hat{\mathbf{c}}_{\alpha \beta}^{a b}=\hat{\mathbf{c}}_{\alpha \beta}^{a b}(k=0), \quad \mathbf{g}_{\alpha \beta}^{a b}=\mathbf{g}_{\alpha \beta}^{a b}\left(\sigma_{\alpha \beta}^{a b}+\right), \quad \partial \mathbf{g}_{\alpha \beta}^{a b}=\frac{\partial \mathbf{g}_{\alpha \beta}^{a b}\left(r=\sigma_{\alpha \beta}^{a b}+\right)}{\partial r}, \\
\frac{\Delta E_{\alpha}^{a}}{V}=2 \pi \rho_{a} \sum_{b} \rho_{b} \sum_{\beta} \int_{0}^{\infty} r^{2} g_{\alpha \beta}^{a b}(r) U_{\alpha \beta}^{(C) a b}(r) d r
\end{gathered}
$$

and

$$
J=-\frac{1}{6} \beta \sum_{a b} \rho_{a} \rho_{b} \sum_{\alpha \beta} \int_{r>\sigma_{\alpha \beta}^{a b}+} g_{\alpha \beta}^{a b}(r) \mathbf{r} \nabla U_{\alpha \beta}^{(C) a b}(r) d \mathbf{r}
$$

The reference system is represented by the multicomponent mixture of uncharged hardsphere chain molecules. Expressions (34), (35) and (36) are quite general and apply for any 
type of the potential outside the hard-core. The quantities, which enter these expressions can be written in terms of the present solution of PMSA for the model at hand. We have

$$
\begin{gathered}
\beta \frac{\Delta E_{\alpha}^{a}}{V}=\beta^{*} \rho_{a} z_{\alpha}^{a} N_{\alpha}^{a}, \quad J=\frac{1}{3} \frac{\Delta E}{V}, \\
\sigma_{\alpha \beta}^{a b} \partial \mathbf{g}_{\alpha \beta}^{a b}=-\mathbf{h}_{\alpha \beta}^{a b}+\sum_{d} \rho_{d} \sum_{\gamma} \sigma_{\alpha \gamma}^{a d} \mathbf{h}_{\alpha \gamma}^{a d} \boldsymbol{\alpha}\left[\mathbf{t}_{\gamma \beta}^{d b}-\left(\tilde{\mathbf{z}}_{\gamma}^{d}\right)^{T} \tilde{\mathbf{a}}_{\beta}^{b}\right]-\mathbf{E} \boldsymbol{\alpha} \sum_{d} \rho_{d} \sum_{\alpha} \lambda_{\gamma \alpha}^{d a} \mathbf{t}_{\gamma \beta}^{d b}- \\
-\frac{1}{2} \sum_{d} \rho_{d} \sum_{\gamma} \sigma_{\gamma}^{d}\left\{\left[\frac{1}{\pi} \mathbf{t}_{\alpha \gamma}^{a d} \boldsymbol{\alpha}-\frac{1}{6}\left(\sigma_{\gamma}^{d}\right)^{2} \mathbf{1}\right] \tilde{\mathbf{E}}^{T} \tilde{\mathbf{A}}_{\beta}^{b}+\left[2 \sigma_{\alpha \gamma}^{a d} \mathbf{g}_{\alpha \gamma}^{a d}-\mathbf{E} \sigma_{\gamma}^{d}-\frac{1}{\pi \sigma_{\gamma}^{d}} \mathbf{t}_{\alpha \gamma}^{a d}\right] \boldsymbol{\alpha} \boldsymbol{\beta}_{\gamma \beta}^{d b}\right\}, \\
\sum_{a b} \rho_{a} \rho_{b} \sum_{\alpha \beta}\left[\boldsymbol{\alpha} \hat{\mathbf{c}}_{\alpha \beta}^{a b} \boldsymbol{\alpha}\right]_{00}=\sum_{a b} \rho_{a} \rho_{b} \sum_{\alpha \beta}\left\{\boldsymbol{\alpha}\left[\left(\mathbf{M}_{\beta \alpha}^{b a}\right)^{T}+\mathbf{M}_{\alpha \beta}^{a b}+\sum_{c} \rho_{c} \sum_{\gamma} \mathbf{M}_{\alpha \gamma}^{a c} \boldsymbol{\alpha}\left(\mathbf{M}_{\beta \gamma}^{b c}\right)^{T}\right] \boldsymbol{\alpha}\right\}_{00},
\end{gathered}
$$

where

$$
\mathbf{M}_{\alpha \beta}^{a b}=-\frac{1}{12}\left(\sigma_{\alpha}^{a}\right)^{3} \tilde{\mathbf{E}}^{T} \tilde{\mathbf{A}}_{\beta}^{b}-\frac{1}{2}\left(\sigma_{\alpha}^{a}\right)^{2} \boldsymbol{\beta}_{\alpha \beta}^{a b}+\sigma_{\alpha}^{a} \mathbf{t}_{\alpha \beta}^{a b}+\lambda_{\beta \alpha}^{b a}\left(\tilde{\mathbf{z}}_{\alpha}^{a}\right)^{T} \tilde{\mathbf{a}}_{\beta}^{b}
$$

Here expression (41) is obtained by differentiating of the equation (8) twice and considering the limit of $r \rightarrow \sigma_{\alpha \beta}^{a b}+$, and expression (42) follows from the first of the equations (25) at $k \rightarrow 0$.

\section{RESULTS AND DISCUSSION}

To illustrate the above solution of the PMSA in this section we present the numerical results for thermodynamical properties of the several versions of charged chain fluid model. We consider two-component mixture of chain polyions with the beads of equal charge $z_{\alpha}^{p}=$ $z_{p}=-1$ and oppositely charged counterions $z_{\alpha}^{c}=z_{c}=1$ (model M1) and two versions of three-component mixture of chain polyions with diblock and alternating distribution of oppositelly charged beads, $z_{+}^{p}=-z_{-}^{p}=1$, and two types of counterions with opposite charges $z_{+}^{c}=-z_{-}^{c}=1$ (models M2 and M3). The densities of the counterions of these two

models were choosed to be $\rho_{+}^{c}=\rho_{-}^{c}=\frac{1}{2} m_{p} \rho_{p}$. Here the indices $p$ and $c$ denote polyion and counterion, respectively. We also consider the one-component version of charged chain fluid 
models with diblock and alternating distribution of the charge along the chain backbone. In all cases the hard-sphere diameters of the chain beads $\sigma_{\alpha}^{p}$ and counterions $\sigma_{\alpha}^{c}$ were choosed to be equal, $\sigma_{\alpha}^{p}=\sigma_{\alpha}^{c}=\sigma=1$. Schematic representation of the models studied are shown in figure 1. Thermodynamical properties of the uncharged versions of these models were calculated using TPTD theory of Chang and Sandler [14.

In figure 2 we compare osmotic coefficient results obtained using the present version of the PMSA, von Solms and Chiew version of PMSA [10 and molecular dynamic (MD) computer simulation method 23] for the model M1 at a different chain length $\left(m_{p}=16,32\right.$ and 64) and Bjerrum length $\lambda_{B}=\beta^{*}=0.833$. In the region of the intermediate densities our theory underestimate and the theory of von Solms and Chiew overestimate the value of the osmotic coefficient $\phi=\beta P / \rho$, where $\rho=\sum_{a} \rho_{a}$. In the region of the low densities both theories predict for the osmotic coefficient its ideal gas value of $\phi=0$, while MD simulation gives the value, which is slightly above 1 . This disagreement could be due to the uncertainties of the MD simulation in the diluted region. In general predictions of the present approach are more accurate than those of von Solms and Chiew approach [10].

In figures 3-5 we compare the density dependence of the osmotic pressure and osmotic coefficient at different values of Bjerrum length $\left(\lambda_{B}=0.833,2.499\right)$ for different polyelectrolyte models. In the case of low Bjerrum length $\left(\lambda_{B}=0.833\right)$ one can see the linear dependence of the osmotic pressure with respect to the density on the $\log -\log$ scale (figures 3a-5a). In this region the osmotic pressure is almost independent of the chain length and distribution of the charge along the chain. With the increase of $\lambda_{B}\left(\lambda_{B}=2.499\right)$ there is a departure from the linearity, which is substantial for the model M1, slightly smaller for the model M2 and almost negligible for the model M3. At the same time one can see the substantial chain length dependence of the osmotic pressure in the case of the model M2. This dependence becomes smaller in the case of the model M1 and is absent for the model M3 (figures 3b-5b). Thus for the systems with more random distribution of the charge along the chain backbone the chain length dependence is smaller. With the increase of the chain length this dependence becomes weaker and it is reasonable to expect, that for $m_{p}>64$ it 
will be negligible for all the models studied.

In figur 6 the liquid-gas phase diagrams for the one-component version of the models with diblock and alternating distribution of the charge are presented. We consider the systems with molecular chain length $m_{p}=2,4,6,8,10$. Computer simulation results are available only in the case of the chains length $m_{p}=2$ [26]. In general theoretical results are in qualitative agreement with computer simulation results with certain disagreement for the slope steepness of the liquid branches of the coexistence curves; the slope of theoretical curve is steeper than that of the computer simulation curve. In addition, the position of the theoretical critical point is shifted towards lower values of the density and higher values of the temperature. According to the previous discussion the systems with diblock distribution of the charge have larger degree of nonideality in comparison with the systems with alternating distribution of the charge. This is reflected in the figure 6 , where the critical temperature of the systems with diblock chains is higher than that of the systems with alternating chains of the same length. The corresponding values of the critical densities is larger in the case of systems with alternating chains. In both cases increase of the chain length leads to the increase of the critical temperature and slight decrease (increase) of the critical density for diblock (alternating) distribution of the charge. We note in passing, that due to the lack of the solution of the equation for screening parameter $\Gamma$ (21) at lower densities we were not able to determine the coexistance curve for the systems with alternating chain charge of the chain length $m_{p}=8,10$ below the temperatures shown in figure $6 \mathrm{~b}$.

Finally in figure 7 we present the liquid-gas phase diagram for the system of trimers with the middle bead charge $z_{2}^{p}=-2 z_{1}^{p}=-2 z_{3}^{p}$, where $z_{1}^{p}$ and $z_{3}^{p}$ are the charges of the terminal beads, $z_{1}^{p}=z_{3}^{p}=1$. Similar as in the case of alternating charged chain system with $m_{p}=8,9$ there is no solution of the equation (21) in the low density region, therefore we were not able to calculate the coexistence curve for the temperatures lower than those shown in the figure. This model can be seen as a complete association limit of the primitive 2:1 electrolyte model. Recent computer simulation studies [27,26,28,29] of the electrolyte primitive model suggest that in the vicinity of the coexistance region the system is highly associated and 
fraction of charged clusters, including free ions, is negligible. Therefore the equilibrium properties of the system are determined by the presence of the neutral ionic clusters, to which the lower order clusters give the main contribution. Assuming that in the case of the electrolyte restricted primitive model (RPM) all such clusters are represented by the neutral ionic pairs the RPM liquid-gas phase diagram has been successfully reproduced by the Monte Carlo (MC) computer simulation method [26] and theoretically [9]. Making similar assumption in the case of the 2:1 primitive electrolyte model the corresponding liquid-gas phase diagram can be modeled using the phase diagram obtained for trimers. Unfortunately computer simulation predictions for the entire phase diagram of the 2:1 electrolyte model currently is not available. Recently Camp and Patey [29] present MC estimate for the critical temperature, which appeares to be $T_{c}^{*}=0.1 \pm 0.01$. The rough estimate of the ranges for the critical density can be obtained from the figure 5 of [29]; our estimate is $\rho_{c}^{*}=0.115 \pm 0.085$. This estimate for the critical point together with Debye-Hückel (DH) theory estimate [29] and MSA phase diagram are shown in figure 7. In addition, for the sake of completeness, we present the liquid-gas phase diagram for the RPM of electrolyte, obtained from the MC simulation method [27,30], MSA and our PMSA for neutral diatomics [9]. One can see that predictions of the PMSA is much better in comparison with usual MSA. In both cases $\mathrm{DH}$ predictions for the critical temperature is close to those of the PMSA, however the corresponding predictions for the critical density are much worse.

\section{CONCLUDING REMARKS}

In this study we obtain an analytical solution of the ideal chain PMSA for the multicomponent mixture of charged hard-sphere flexible linear chain molecules. This solution apply to any mixture of chain molecules with arbitrary distribution of the charge and size of the beads along the molecular backbone. We present closed form analytical expressions for thermodynamical and structure properties of the system. These expressions are used to calculate thermodynamics of several different versions of charged hard-sphere chain model. 
We consider two-component mixture of chains with equally charged beads and oppositely charged counterions, two versions of the three-component mixture of chains with diblock and alternating distribution of the charge and two types of oppositely charged counterions. Comparison of the theoretical predictions for the osmotic pressure of the two-component model with corresponding computer simulation predictions shows that the present version of the ideal chain PMSA theory is reasonably accurate and gives somewhat better agreement than that of the theory of von Solms and Chiew [10]. Effects due to the difference in Bjerrum length, molecular chain length and distribution of the charge was also studied. The largest deviation from ideal behaviour was demonstrated by the system with uniform distribution of the charge; behaviour of the system with alternating distribution of the charge shows much less nonideality. This conclusion is varyfied by the comparison of the liquid-gas phase diagrams calculated for the one-component version of the models with diblock and alternating distribution of the charge; the latter model has substatially lower critical temperature than that of the former. With the increase of the chain length the critical temperature for both models increases, while the corresponding critical density slightly increases for alternating charge model and decreases for diblock charge model. Finally it was demonstrated, that liquid-gas phase diagram of the neutral trimers with doubly charged middle bead and singly charged terminal beads of the opposite sighn can be used to correct MSA results for the phase diagram of 2:1 primitive electrolyte model.

\section{ACKNOWLEDGMENTS}




\section{REFERENCES}

[1] M. Dymitrowska, and L. Belloni, J. Chem. Phys. 111, 6633(1999).

[2] K. S. Schweizer, and J. G. Curro, Adv. Chem. Phys. 98, 1(1997).

[3] J. W. Jiang, Y. L. Liu, Y. Hu, and J. M. Prausnitz, J. Chem. Phys. 108, 780(1998).

[4] J. Jiang, H. Liu, and Y. Hu, J. Chem. Phys. 110, 4952(1999).

[5] G. Stell, and Y. Zhou, J. Chem. Phys. 91, 3618(1989); Y. Zhou, and G. Stell, ibid 96, 1504(1992); 96, 1507(1992); 102, 8089(1995).

[6] Yu. V. Kalyuzhnyi, and G. Stell, Chem. Phys. Lett. 240, 157(1995).

[7] L. Blum, Yu. V. Kalyuzhnyi, O. Bernard, and J. N. Herrera-Pacheco, J. Phys.: Cond. Matter 8, A143(1996).

[8] I. A. Protsykevytch, Yu. V. Kalyuzhnyi, M. F. Holovko, and L. Blum, J. Mol. Liquids $\mathbf{7 3 , 7 4}, 1(1997)$.

[9] Yu. V. Kalyuzhnyi, Mol. Phys. 94, 735(1998).

[10] N. von Solms, and Y. C. Chiew, J. Chem. Phys. 111, 4839(1999).

[11] M. S. Wertheim, J. Stat. Phys. 42, 459; ibid 42, 477(1986).

[12] M. F. Holovko, and Yu. V. Kalyuzhnyi, Mol. Phys. 73, 1145(1991).

[13] J. Chang, and S. I. Sandler, J. Chem. Phys. 102, 437(1995).

[14] J. Chang, and S. I. Sandler, J. Chem. Phys. 103, 3196(1995).

[15] Yu. V. Kalyuzhnyi, and P. T. Cummings, J. Chem. Phys. 104, 3325(1996).

[16] P. J. Rossky, and R. A. Chiles, Mol. Phys. 51, 661(1984).

[17] D. Chandler, R. Silbey, and Ladanyi, Mol. Phys. 46 1335(1982).

[18] Yu. V. Kalyuzhnyi, and G. Stell, Mol. Phys. 78, 1247(1993). 
[19] G. Stell, Physica a 231, 1(1996).

[20] Yu. V. Kalyuzhnyi, and P. T. Cummings, J. Chem. Phys. 105, 2011(1996).

[21] Yu. V. Kalyuzhnyi, C.-T. Lin, and G. Stell, J. Chem. Phys. 108, 6525(1998).

[22] J. S. Høye and G. Stell, J.Chem.Phys. 67, 439(1977).

[23] M. J. Stevens, and K. Kremer, J. Chem. Phys. 103, 1669(1995).

[24] O. Bernard, and L. Blum, J. Chem. Phys. 112, 7227(2000).

[25] L. Blum, Mol. Phys. 30, 1529(1975); L. Blum, and J. S. Høye, J. Phys. Chem. 81, 1311(1977).

[26] J. C. Shelley, and G. N. Patey, J. Chem. Phys. 103, 8299(1995).

[27] A. Z. Panagiotopoulos, Fluid Phase Equil. 76, 97(1992).

[28] J.-M., Caillol, and J.-J. Weiss, J. Chem. Phys. 102, 7610(1995).

[29] P. J. Camp, and G. N. Patey, J. Chem. Phys. 111, 9000(1999).

[30] J. M. Caillol, J. Chem. Phys. 100, 2161(1994). 


\section{CAPTIONS TO THE FIGURES}

Figure 1. Schematic diagrams of the models with uniform distribution of the charge (M1), diblock distribution of the charge (M2) and alternating distribution of the charge.

Figure 2. Osmotic coefficient $\phi=P V / N k T$ as a function of the packing fraction $\eta=\pi \rho \sigma^{3} / 6$ for the model M1 at $\lambda_{B}=0.833$ for $m_{p}=16$ (a), $m_{p}=32$ (b) and $m_{p}=64$ (c). Predictions of the present theory (solid lines), von Solms and Chiew theory [10] (dashed lines) and computer simulation predictions [23] (diamons).

Figure 3. Osmotic pressure $P^{*}=\beta P \sigma^{3}$ (a) and osmotic coefficient $\phi=P V / N k T$ (b) as a function of packing fraction $\eta=\pi \rho_{T} \sigma^{3} / 6\left(\rho_{T}=\sum_{a} m_{a} \rho_{a}\right.$ for the model M1 from the present theory at $\lambda_{B}=2.499$ (solid lines), $\lambda_{B}=0.833$ (dashed lines) and $\lambda_{B}=0$ (dashed-dotted lines). For each set of lines from the top to the bottom at $\log \eta=-2$ $m_{p}=8,16,32,64$. Symbols are computer simulation predictions [23] for $\lambda_{B}=0.833$ and for $m_{p}=16$ (diamonds), $m_{p}=32$ (circles) and $m_{p}=64$ (squares).

Figure 4. The same as in figure 3 for the model M2.

Figure 5. The same as in figure 3 for the model M3.

Figure 6. Liquid-gas phase diagram in $T^{*}=1 / \beta^{*}$ versus reduced density of the beads $\rho_{b}^{*}=m_{p} \rho \sigma^{3}$ coordinates for one component chain fluids with diblock distribution of the charge (a) and with alternating distribution of the charge (b). From the top to the bottom at $\rho_{b}^{*}=0.05 m_{p}=10,8,6,4,2$. Solid lines are predictions from the present theory and diamonds are computer simulation predictions [26] for $m_{p}=2$.

Figure 7 Liquid-gas phase diagram in $T^{*}=1 / \beta^{*}$ versus reduced ionic density $\rho^{*}=$ $\left(\rho_{+}+\rho_{-}\right) \sigma^{3}$ coordinates for restricted primitive model (RPM) of electrolyte (lower portions of the figure for $\left.T^{*}>0.08\right)$ and for the primitive model (PM) of electrolyte with 2:1 assymetry in charge (upper portions of the figure for $T^{*}<0.08$ ). Present theory (solid lines), MSA (dashed lines), Debye-Hückel theory [29] (squares), computer simulation estimate for the critical point of 1:2 electrolyte PM [29] (open diamonds) and for the phase diagram of 
electrolyte RPM (open circles [27], solid diamonds [30]). 


\section{FIGURES}

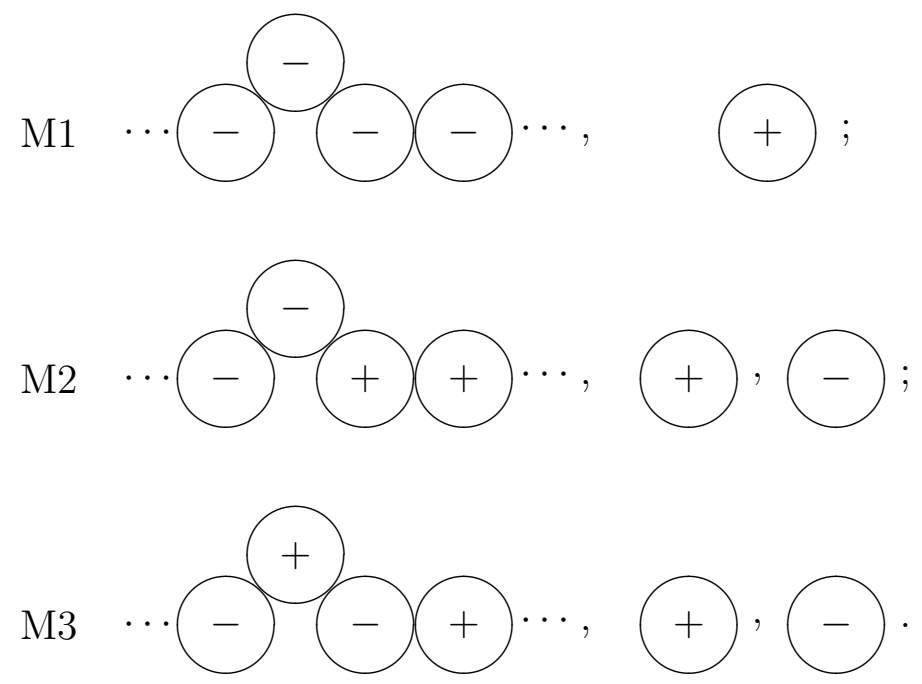

Figure 1 (Kalyuzhnyi and Cummings) 

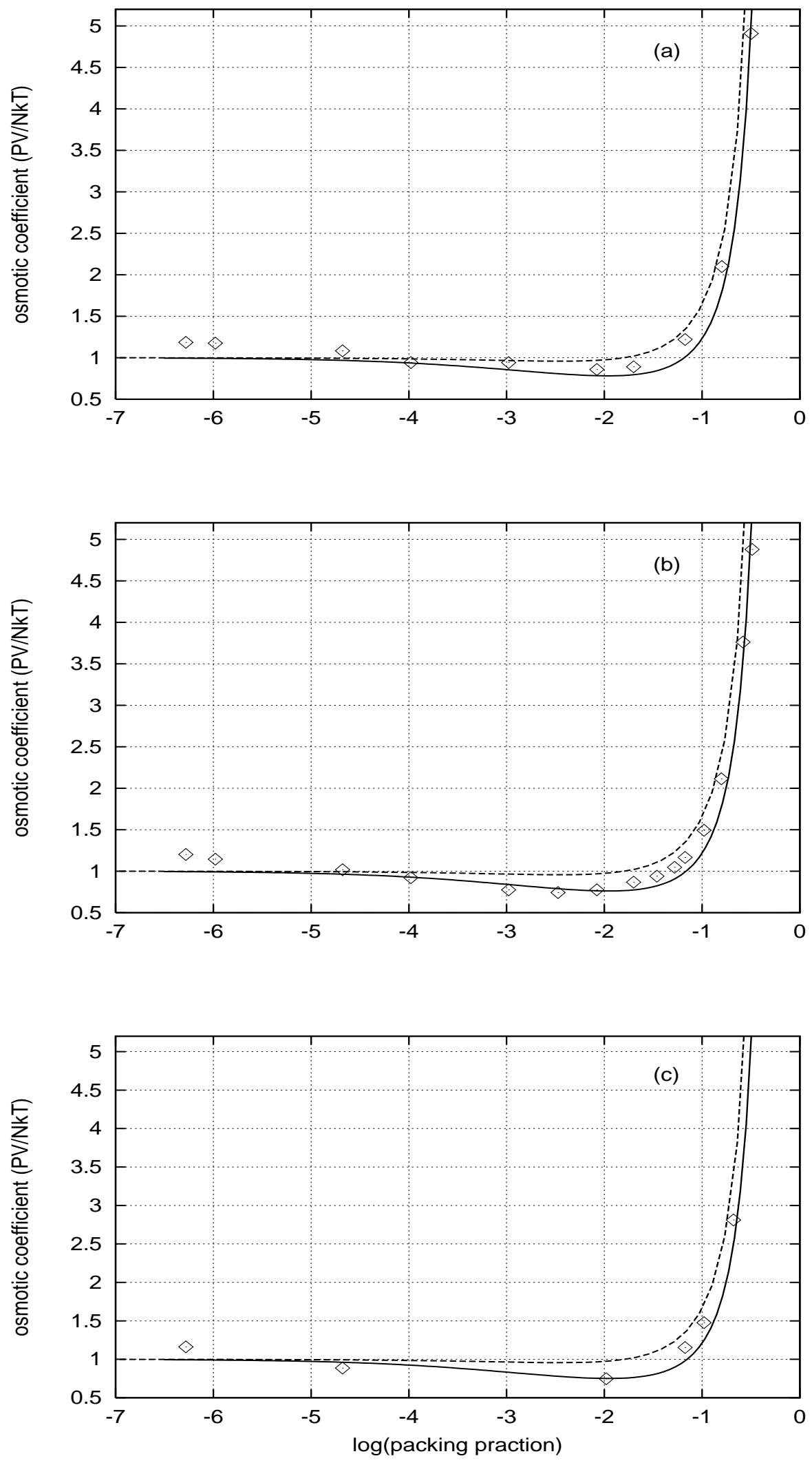

Figure 2 (Kalyuzhnyi and Cummings) 

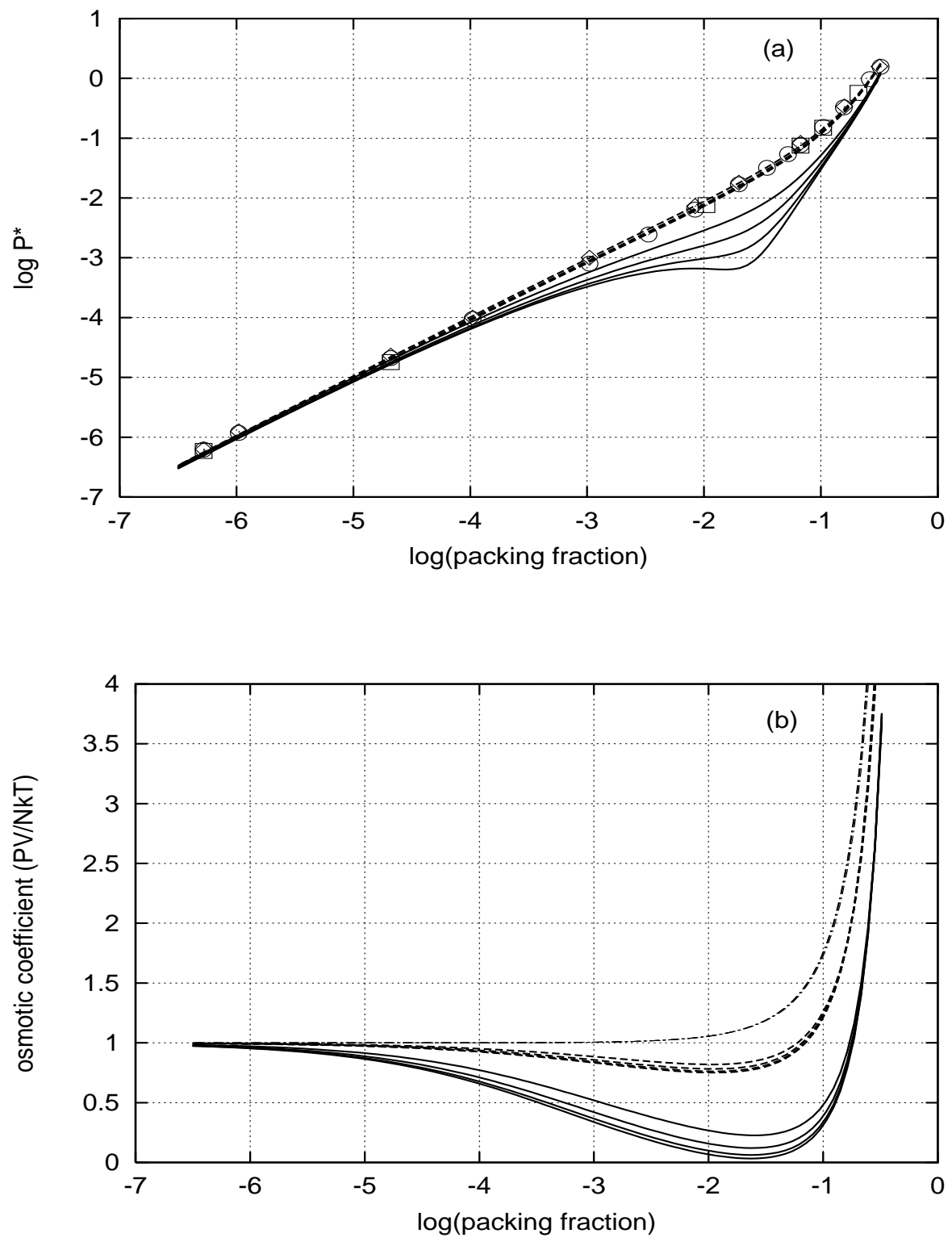

Figure 3 (Kalyuzhnyi and Cummings) 

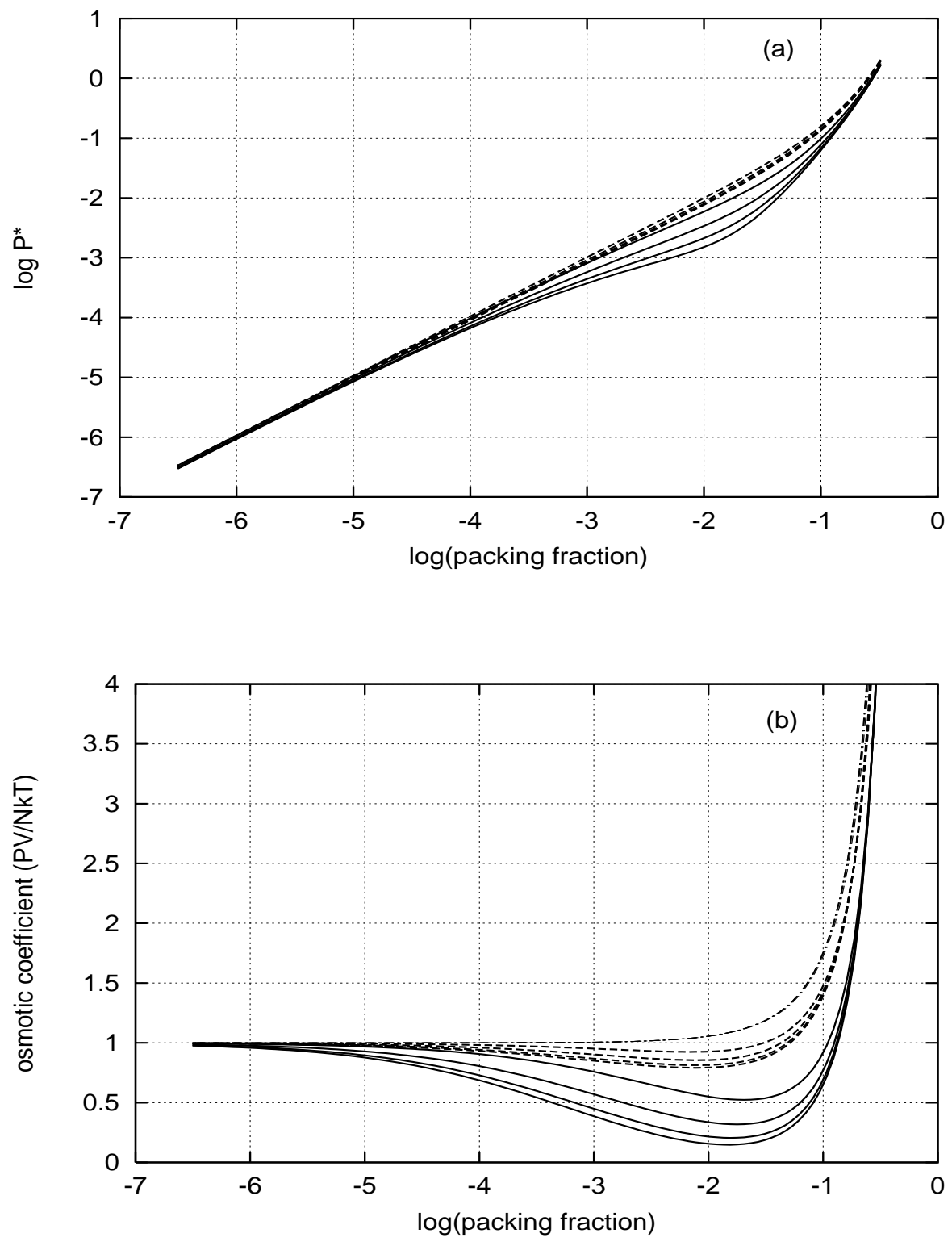

Figure 4 (Kalyuzhnyi and Cummings) 

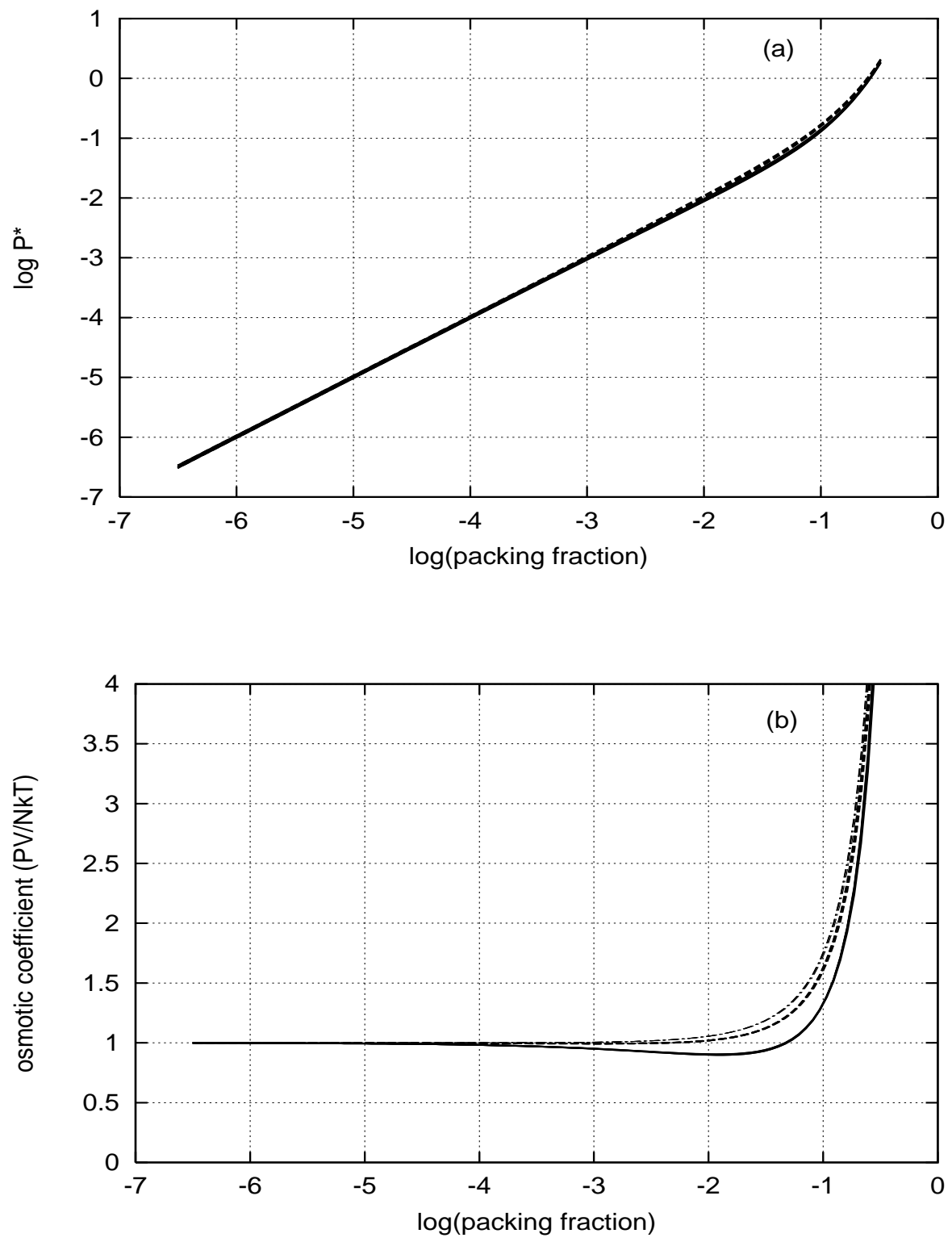

Figure 5 (Kalyuzhnyi and Cummings) 

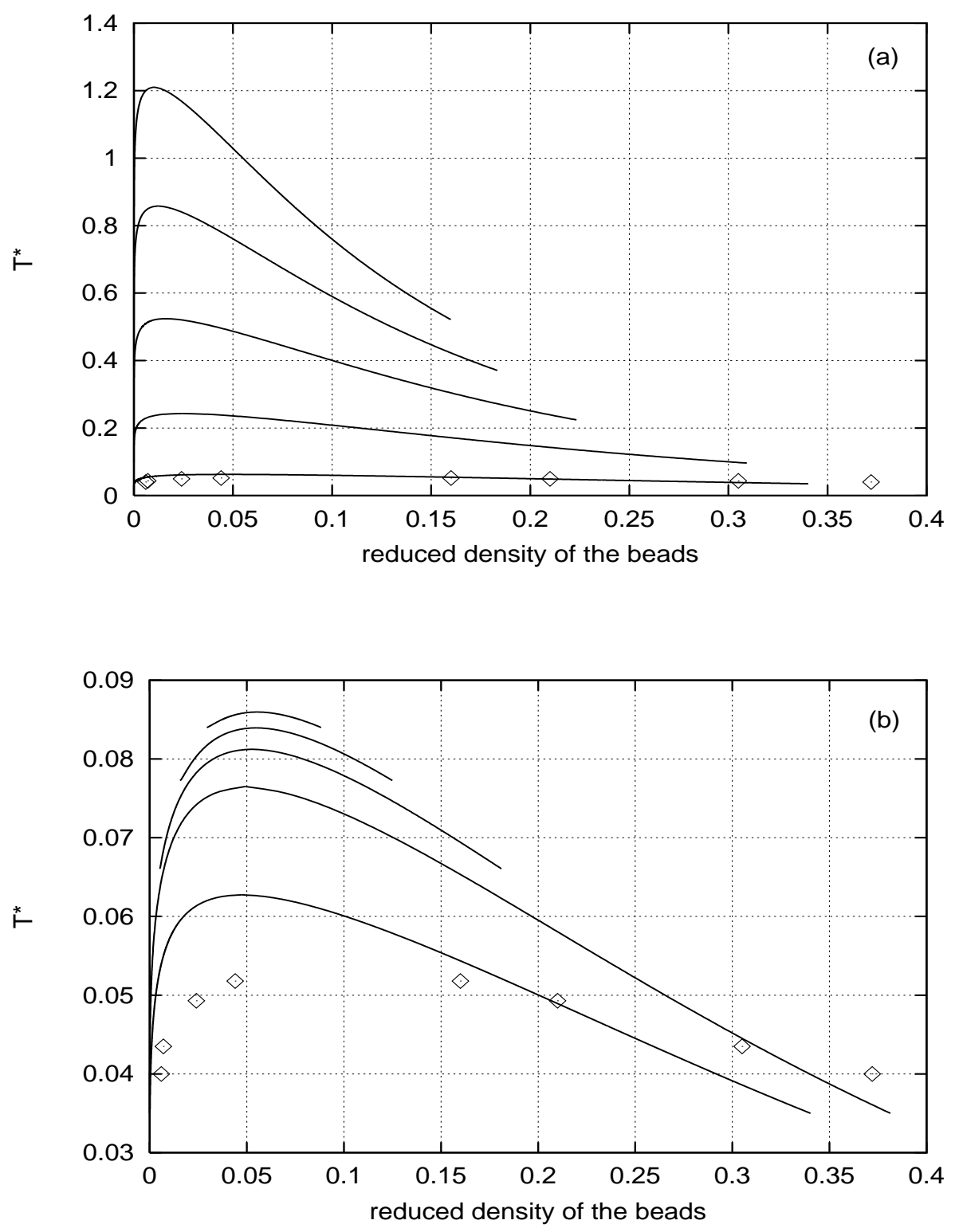

Figure 6 (Kalyuzhnyi and Cummings) 

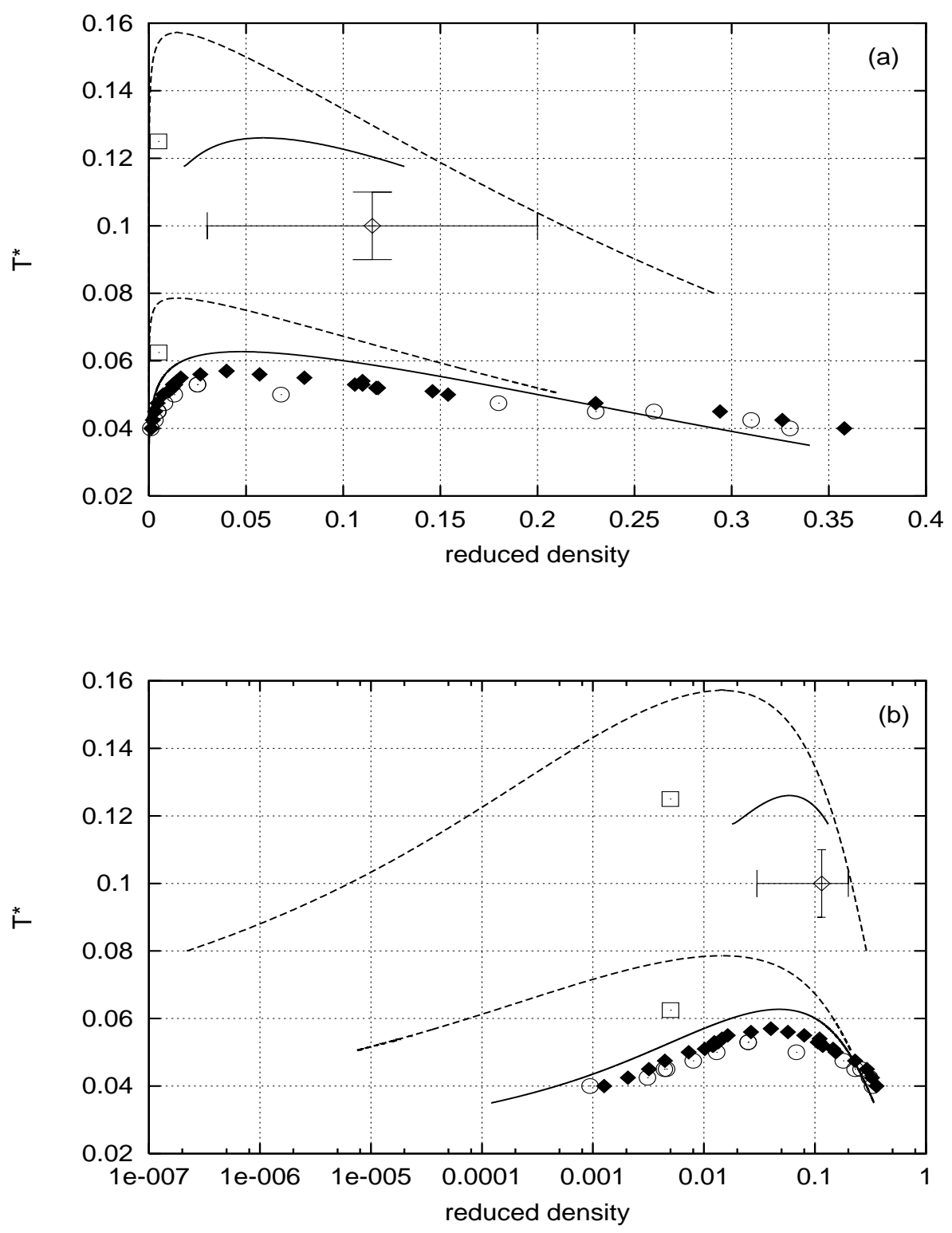

Figure 7 (Kalyuzhnyi and Cummings) 